\title{
El impacto internacional de la ley Helms-Burton ${ }^{1}$
}

\author{
Joaquín Roy
}

La llamada ley Helms-Burton ha atraido la atención no solamente de los especialistas en Derecho Internacional, sino también de los politólogos y los analistas de las relaciones internacionales. Las expectativas de duros litigios ante los tribunales (que se suponía podrían llegar hasta la Corte Suprema de los Estados Unidos), de momento no se han visto confirmadas, a causa de la suspensión temporal por el presidente Clinton del título III, que permitiría a los antiguos propietarios de bienes confiscados en Cuba que demandaran a los que "traficaran" con ellas. El transitorio compromiso firmado entre Washington y Bruselas congeló el proceso de enfrentamiento transatlántico y el alivio del daño infligido a la $\mathrm{OMC}$, aunque las medidas adicionales del Congreso de los Estados Unidos amenazan con mantener la tensión y con ella la latente posibilidad de litigios judiciales. Como legado, dos dimensiones se mantienen como firmes candidatas para el análisis, todavía abierto a los especialistas. En primer lugar, se destaca el impacto ejercido por las motivaciones politicas de la ley en las relaciones internacionales de los Estados Unidos con el resto del mundo; en segundo lugar, el tema de la ley Helms-Burton seguirá atrayendo la atención de los juristas por la complejidad de sus medidas, las interpretaciones sobre su constitucionalidad, o la aparente violación de las normas internacionales. En este trabajo se presta especial atención a esta segunda dimensión, en íntima relación con la primera.

\footnotetext{
'Este estudio originariamente fue presentado en la conferencia de la European Community Studies Association (ECSA), celebrada en Seattle, Washington, del 27 de mayo a 1 de junio de 1997. Con posterioridad, se ofreció como conferencia en el departamento de Derecho Internacional de la Facultad de Derecho de la Universitat Pompeu Fabra de Barcelona, el $1^{\circ}$ de julio de 1997. Debo mi agradecimiento por diversas contribuciones a Ambler H. Moss, Oriol Casanovas, Angel Viñas, IRELA, el Consulado de México en Miami, la Comisión Europea, la Oficina de Información Diplomática del Ministerio de Asuntos Exteriores de España y la Fundación CIDOB por los materiales suministrados. Especialmente, dejo constancia del interés generosamente demostrado por Enrique Baloyra poco antes de su fallecimiento el 26 de julio de 1997.
} 


\section{Origen y desarrollo.}

El 11 de abril de 1997, la Comisión Europea, representada por su vicepresidente segundo, el comisario británico Leon Brittan como responsable de asuntos de comercio exterior, y el Gobierno de Estados Unidos, representado por el Subsecretario de Estado para el Comercio Exterior, Stuart Eizenstat, acordaron un compromiso que neutralizaba temporalmente la ley Helms-Burton, apenas un año después de haberse aprobado. De ejecutarse fielmente los puntos más importantes del acuerdo (la Unión Europea retiraría su demanda interpuesta en la Organización Mundial del Comercio, mientras los Estados Unidos congelarían la aplicación efectiva de la ley), se habría puesto fin a uno de los episodios más serios de enfrentamiento entre Washington y Bruselas, al igual que importante diferendo entre los Estados Unidos y sus socios en el TLC.

El balance final revela, por una parte, que los principales autores y patrocinadores de la ley parecían no demostrar preocupación alguna por las repercusiones internacionales de la legislación; los objetivos locales y parlamentarios primaban. Por otro lado, no se percibía un movimiento de aminorar la marcha por la senda de la confrontación que los socios comerciales de los Estados Unidos habían tomado en Europa y en el continente americano, cuando constataban que sus legítimos intereses corrían serios riesgos. La ley Helms-Burton se interpretaba como una grave violación de las convenciones internacionales, básicamente en 10 que respecta al concepto de extraterritorialidad.

\section{Contexto de la génesis.}

El objetivo de la "Ley de la Libertad y la Solidaridad con Cuba", versión en español de la oficialmente denominada "Cuba Liberty and Democratic Solidarity (Libertad) Act of 1996", pero popularmente aludida como "Ley Helms-Burton"2, ha sido fundamentalmente político. Medianie la presión para desalentar las inversiones extranjeras en Cuba (a través de la amenaza de demandas judiciales y las restricciones de viaje a los Estados Unidos), ha perseguido provocar un mayor deterioro económico que acelerara el final del régimen dictatorial cubano. Esta ley es uno de los últimos eslabones del empleo de los métodos económicos en el entramado de la política exterior de los Estados Unidos. En este sentido, Washington se ha destacado entre las diferentes potencias, ya

\footnotetext{
${ }^{2}$ Congreso de los Estados Unidos, PubI., No104-114, 110 Stat.
} 
que ha protagonizado o intervenido en 77 de los 116 casos de sanciones económicas documentadas en el último medio siglo, con una proporción creciente: entre 1954 y 1963 lo hizo en el 50\% de los casos; en el $70 \%$ entre 1964 y 1973 , y en el $78 \%$ entre 1974 y el 1994.3

$\mathrm{El}$ origen legislativo reside en la llamada "ley Torricelli" y otros instrumentos legales anteriores. Su desarrollo se extiende desde su proposición como proyecto de ley (bill), e incluye la aprobación legislativa, la subsecuente ratificación presidencial de 12 de marzo de 1996, y la provisional suspensión (en tres períodos sucesivos y renovables de seis meses) por el propio presidente Bill Clinton del controvertido título III.

\section{Desarrollo legal y contexto político.}

La ley Helms-Burton es la culminación no solamente de la evolución de la actitud política de los Estados Unidos hacia Cuba, sino que también tiene su propia cronología en la dimensión jurírica. ${ }^{4}$ Desde el triunfo de la Revolución Cubana, todas las acciones legales norteamericanas han sido una reacción sucesiva a la inexorable inclinación del régimen cubano hacia la órbita política y económica de la Unión Soviética en 1960. El año anterior, una serie de medidas del gobierno cubano (siempre perjudiciales para los intereses norteamericanos) había preparado el terreno para la confrontación: Ley 78 de Recuperación de Activos Malversados de 13 de febrero, Ley 122 de Reducción de Rentas de 10 de marzo, Ley 218 de Terrenos Baldíos de 17 de abril, Ley de Reforma Agraria de 17 de mayo, Ley (617) Minera de 27 de octubre, Ley 635 del Petróleo de 20 de noviembre, y Ley 647 del Trabajo de 24 de noviembre. 5

Tras los acuerdos comerciales firmados el 13 de febrero de $1960, \mathrm{La}$ Habana y Moscú procedieron a intercambiar azúcar por petróleo, además de que Cuba comenzaba a beneficiarse con créditos y desarrollo tecnológico. Como respuesta, el presidente Eisenhower redujo progresivamente la cuota de azúcar ( $70 \%$ de todas exportaciones cubanas) que Estados Unidos importaba de Cuba (según la Sugar Act de 1948, a un $80 \%$ del precio del mercado abierto) y el 6 de julio de 1960, a través del American Sugar, Bill, la eliminó completamente. Como represalia, Cuba

\footnotetext{
${ }^{3}$ Ver el artículo de Daniel Sabbach, mencionado en la bibliografia.

${ }^{4}$ Para un repaso de la interconexión de los aspectos jurídicos con los politicos, se recomiendan los trabajos de los siguientes autores, cuyos datos completos se incluyen en la bibliografia: Bar Association of the City of New York, Bourque, Cain, Donner, Porotsky, Wong y Zipper.

${ }^{5}$ Para una cronología comparativa, véase el anexo del artículo de Archibald Ritter, incluido en la bibliografia.
} 
comenzó a nacionalizar las propiedades norteamericanas. Las respuestas de Estados Unidos no se hicieron esperar. Washington ya había suspendido las relaciones diplomáticas el 3 de enero de 1961 y había intentado el derrocamiento de Castro mediante la operación de Bahía Cochinos de abril. Esta vez la acción norteamericana consistió en la imposición en octubre de un embargo económico limitado a bienes norteamericanos. El 3 de febrero de 1962 el presidente John F. Kennedy se basó en dos leyes, la Foreign Assistance Act de 1961 y la Trading with the Enemy Act (aprobada en 1917 en el marco de la Primera Guerra Mundial), para decretar por la Presidential Proclamation 3447 un embargo de todo comercio con Cuba. Por su parte, el Departamento del Tesoro promulgó el 8 de julio de 1963 las medidas llamadas Cuban Assets Control Regulations por las que se prohibía a los norteamericanos cualquier relación comercial y financiera con Cuba, con la excepción de las actividades de las compañías subsidiarias.

La escalada del enfrentamiento se traspasó a los foros interamericanos, principalmente la Organización de los Estados Americanos (OEA), con el resultado de la ruptura de vínculos por parte de todos los países latinoamericanos, a excepción de México, como consecuencia de la crisis de los misiles de octubre de 1962 y la injerencia cubana en América Latina. Después de un período de cierta normalidad, ante las aventuras cubanas en Africa en la década de los setenta, los Estados Unidos impusieron serios límites a los viajes a Cuba. En los ochenta, el presidente Ronald Reagan aplicó mayores restricciones encaminadas a obstaculizar que el gobierno cubano pudiera negociar libremente la deuda externa, al tiempo que presionaba a las compañías norteamericanas en el exterior para que eliminaran sus relaciones indirectas con Cuba a través de las subsidiarias. ${ }^{6}$

Como adelanto de los cambios que se avecinaban, Cuba comenzó a favorecer legalmente los intercambios económicos y las inversiones. En 1982 se aprobó una ley para regular las actividades de los consorcios, ${ }^{7}$ al tiempo que ofrecía posibilidades para el arrendamiento de propiedades por largos plazos, en condiciones casi parecidas a los derechos de propiedad. Las regulaciones laborales fueron también modificadas en 1990 para facilitar el establecimiento de empresas turísticas. Finalmente, en 1992 algunos fragmentos de la Constitución (arts. 14, 15 y 18) se

\footnotetext{
${ }^{6}$ Entre otras fuentes, se recomienda la consulta del trabajo de Donna Rich Kaplowitz mencionado en la bibliografia.

${ }^{7}$ Decreto Legislativo ${ }^{\circ} 50,15$ de febrero de 1982.
} 
adaptaron para suavizar la intransigencia contra la propiedad privada. Lenta, pero inexorablemente, los vínculos comerciales de Cuba se reajustaron. Mientras en 1989 solamente el 5,7\% de sus intercambios comerciales se hacían con las Américas, en 1994 el continente había ya ocupado el 34,7\%; mientras el $87,7 \%$ de su comercio era en 1989 con la Europa del Este, el $45 \%$ ya se hacía principalmente con la Europa Occidental en 1994. Ante los aparentes éxitos comerciales de Cuba, y la posibilidad de lograr una evolución económica (al modo chino, sin satisfacer el peaje de la reforma política), la respuesta norteamericaná no $s \in$ hizo esperar.

En plena campaña electoral para las elecciones presidenciales, el 23 de octubre de 1992 el presidente Bush aprobó la Cuban Democracy Act. Se trataba de un proyecto de ley presentado en abril del mismo año por el diputado de Nueva Jersey Robert Torricelli, y que también había sido avalado por el ex-gobernador de Arkansas y entonces candidato presidencial Bill Clinton. La vuelta de tuerca estaba esta vez representada por la amenaza de sanciones contra las compañías norteamericanas que mantuvieran tratos con Cuba a través de subsidiarias; también prohibía que los buques que hubieran recalado en Cuba atracaran luego en puertos de los Estados Unidos. Sin embargo, esta ley concedía al poder ejecutivo la opción de ajustarla con "respuestas sopesadas" (calibrated responses) en forma de levantamiento parcial de sanciones a cambio de acciones positivas por parte de Cuba.

Tras la victoria republicana en las elecciones legislativas de noviembre de 1994, el senador Jesse Helms se convirtió en Presidente de la Comisión de Relaciones Exteriores de la cámara alta norteamericana. Inmediatamente, anunció que el espíritu de la "ley Torricelli" sería endurecido y se transformaría en la "Ley de la Libertad y la Solidaridad con Cuba", por la cual las compañías que operaban en los Estados Unidos y que estaban comerciando con intereses norteamericanos expropiados en Cuba podían verse sujetas a demandas. Este esquema adquirió visos más agudos cuando, tras el incidente del derribo de las avionetas de la organización "Hermanos al Rescate", el presidente Clinton aprobó la ley Helms-Burton el 12 de marzo de $1996 .{ }^{8}$

Mientras el título I, sección 102, codifica todas las anteriores reglas y órdenes gubernamentales, y convierte al Congreso (en lugar del Presidente) en la clave de cambios futuros, esta ley básicamente pretende ajustar el embargo mediante los siguientes mecanismos:

\footnotetext{
${ }^{8}$ Para un repaso de la interpretación de las motivaciones de todas las partes involucradas, se recomienda la lectura del ensayo de William Leogrande mencionado en la bibliografia.
} 
- La sección 103 prohibe préstamos, créditos o financiamiento por parte de ciudadanos o residentes de los Estados Unidos en transacciones de propiedades confiscadas;

- la sección 104 obliga al gobierno de los Estados Unidos a votar contra la admisión de Cuba en el FMa y' el Banco Mundial, entre otras instituciones financieras, hasta que no resurja la democracia en Cuba;

- el título III permite que los ciudadanos de los Estados Únidos presenten demandas en Jos tribunales norteamericanos contra cualquiera que "trafique" en propiedades confiscadas por Cuba y extiende este derecho a las personas que no eran ciudadanos en el momento de dicha confiscación;

- el título IV deniega la admisión en los Estados Unidos de ciudadanos extranjeros (dirigentes de empresas, sus familias, o accionistas) que estén involucrados en "traficar" con propiedades confiscadas.

\section{Análisis jurídico.}

La ley ha generado una considerable energía en la comunidad jurídica. Los más beneficiados parecen ser los abogados que fueron inmediatamente contratados $u$ ofrecieron sus servicios a las personas o empresas potencialmente aquejadas de la aplicación de la ley o a los intereses que podían verse encuadrados en la categoría de demandantes. ${ }^{9}$ Esta legislación también ha alentado el interés de catedráticos y diversos expertos en derecho internacional que han elaborado unos notables estudios sobre la constitucionalidad de la ley y sus potenciales violaciones de las normas internacionales. ${ }^{10}$ Los análisis de 10 juristas coinciden en algunas líneas generales que son sumamente críticas, sin que sea posible separar el texto jurídico de su verdadero contexto político. Los aspectos más descollantes pueden agruparse alrededor de tres ejes principales: derecho constitucional, tratados internacionales y procedimiento judicial. En síntesis, los puntos más destacados son los siguientes:

- Prestando especial atención a la posible violación de las leyes fundamentales de los Estados Unidos, se constata que, salvo raras excepciones, ${ }^{11}$ el consenso generalizado en el sector académico es sumamente crítico sobre la constitucionalidad de la ley. Este diag-

\footnotetext{
${ }^{9}$ Véanse los trabajos (hasta la fecha inéditos) de Aitozano, Mallet, Muse y Ruiz Bravo presentados en el Seminario sobre "El refuerzo del embargo de EE.UU. contra Cuba", organizado por IRELA en Sitges, los dias 8-10 de julio de 1996.

${ }^{10}$ Remitimos a los trabajos mencionados en la nota 3.

${ }^{11}$ Especialmente, véanse la ponencia inédita de Sergio Alvarez-Mena y Daniel Crane, y el artículo de Brice Clagett, cuyos datos se detallan en la bibliografia.
} 
nóstico está cimentado por la obviedad consistente en que los objetivos políticos de la ley priman sobre los jurídicos o comerciales. Como otras similares medidas coercitivas de este tipo, se alega que la ley es un ataque (por su sección 102) contra la propia Constitución (5a enmienda) que garantiza la libertad de viaje de los ciudadanos norteamericanos. Asunto diferente (pero paralelo y más controversial en el exterior) es la amenaza de denegación de visado para entrar en los Estados Unidos o trabajar en su territorio a los directivos de las compañías que "trafiquen" con propiedades norteamericanas confiscadas por el gobierno cubano. El argumento que eximen los que apoyan la medida es el derecho que todo Estado tiene de garantizar el control de sus fronteras.

- En lo que atañe a la acción exterior, la ley Helms-Burton revela una meta de política exterior que se impone a los tribunales de los Estados Unidos. Es una violación explícita de la separación de poderes (un tabú americano, compartido por la mayoría de los ordenamientos constitucionales democráticos). Es un caso insólito de automutilación de los privilegios del propio Presidente para liderar la política exterior. Pero el detalle más crucial de la ley es que codifica un detalle transitorio de política exterior: eleva a nivel de ley federal to que antes era reglamentario y obliga al Presidente a contar con el consentimiento del Congreso para modificar las condiciones anteriores del embargo y condiciona su abrogación a la explícita restauración de la democracia en Cuba, la desaparición política del propio Castro y la implantación de la economía de mercado con requisitos muy claros. Aunque el Presidente pueda, como ya lo ha hecho en tres ocasiones (junio de 1996, enero de 1997 y julio de 1997), suspender por períodos de seis meses la ejecución del título III, su carácter de ley la convierte en foco fácil de manipulación e intrincadas manipulaciones políticas. Por ejemplo, en el momento de producirse un cambio de gobierno cubano, pudieran no darse todos los requisitos exigidos en caso de una transición lenta y progresiva, o bien que los detalles no fueran del todo del agrado de los que propusieron o inspiraron la ley.

- Con respecto a la interpretación del derecho de ciudadanía, la ley ha abierto una especie de caja de Pandora en lo que respecta a una nueva definición de ciudadanía que permite a los cubanos nacionalizados en los Estados Unidos disfrutar de la protección estatal por las consecuencias de hechos que tuvieron lugar antes de ser ciudadanos norteamericanos: es un privilegio retroactivo que la ley le concede 
graciosamente, en contraste con la ausencia de la misma oportunidad para los antiguos ciudadanos del resto de los países. Se trata de una invitación a demandas judiciales por agravio comparativo causado por la posible discriminación basada en detalles y argumentos étnicos o de nacionalidad original. Puede ser una contradicción con toda la tradición de los tribunales norteamericanos: la protección legal solamente se puede disfrutar por hechos acaecidos cuando se gozaba de la ciudadanía de los Estados Unidos.

- En el plano de procedimiento judicial, se crea un escenario de confusión legal con el resultado de que el planteamiento de múltiples demandas podría hacer colapsar algunos tribunales norteamericanos con juicios largos e insólitos que podrían culminar en un lógico final anticlimático: la declaración de las máximas instancias sobre la inconstitucionalidad de la ley. Finalmente se cuestiona la declaración de "inaplicabilidad de la Doctrina de Estado" (sección 302) por la que se conmina a que "ningún tribunal de los Estados Unidos podrá abtenerse", ya que es una invitación a un serio enfrentamiento entre los poderes legislativo y judicial.

De todas maneras, la ley estrictamente sólo existe en una dimensión política y potencialmente en el plano económico, pero desde el punto de vista de la tradición norteamericana es papel mojado hasta el momento en que un tribunal no la convierta en verdadera ley. Las perspectivas de que se produzca un trasiego de sumarios a través de los juzgados son notables teóricamente, teniendo en cuenta que los requisitos mínimos ảel título III de la ley permiten que todos los que fueron objeto de expropiaciones superiores a un valor de 50.000 dólares puedan presentar reclamaciones. Eso quiere decir que aproximadamente 400.000 cubanos que ahora son ciudadanos de los Estados Unidos podrían demandar a los que "trafiquen" con sus antiguas propiedades, que ahora están en manos del Estado cubano. En contraste, solamente unas 300 compañías o personas norteamericanas de origen podrían beneficiarse de esta ley (o mejor incluso, de la "Cuba Claims Act" de 1964), porque muchas propiedades ya no tienen ningún valor mercantil. Por ese motivo cínicos comentaristas la bautizaron como "Ley Bacardi", en representación emblemática de importantes intereses expropiados por la Revolución. Este esquema es, sin embargo más complejo.

El apelativo "Bacardi" puede ser erróneamente interpretado como que el proyecto legislativo fue respaldado exclusivamente por grandes intereses de origen cubano. En realidad, las grandes compañías no vieron 
con buenos ojos que el número de posibles demandantes se ampliara, ya que eso representaría disminuir sus posibilidades de recuperar sustanciales sumas. Estos antiguos dueños de entidades confiscadas que ya estaban reconocidos como reclamantes en los años 60 preferían que la lista permaneciera igual, y para ello formaron una sociedad llamada Joint Corporate Committee on Cuban Claims, con residencia legal en Stanford, Conn., compuesta mayoritariamente de las compañías con demandas certificadas y que se opusieron a la evolución de la ley por esa razón. Según la U. S. Foreign Claims Settlement Commission, habría unas 40 compañías norteamericanas con reclamaciones superiores a los $10 \mathrm{mi}-$ llones de dólares. ${ }^{12}$

En la dimensión del entramado de los acuerdos internacionales, la sección 103 (y todo el espíritu de la ley en cuanto al uso de la extraterritorialidad) puede violar el común entendimiento del derecho internacional y diferentes tratados e instrumentos legales internacionales suscritos por los Estados Unidos. Por ejemplo, se destacan los acuerdos del FMI (artículos 8 y 9), el Banco Mundial (artículos 6 y 10), el BID (artículos II y XI), el ordenamiento jurídico del TLC (NAFTA), y el de la Organización Mundial de Comercio. Resumiendo con cierto sentido del humor el sentimiento de la comunidad jurídica, el abogado Muse ${ }^{13}$ aludía que al tratar de ofrecer una alternativa legal para compensar a los que fueron expropiados equivalía a permitir a los automovilistas que quisieran aparcar en lugares señalizados y reservados para esta función para que atropellaran a transeúntes que por allí pasaran o ocuparan brevemente ese espacio.

Por el contrario, los argumentos en defensa de la ley emitidos por los expertos que se inclinan por las tesis del gobierno norteamericano enfocan las dimensiones que resaltan la legalidad constitucional y el cumplimiento de los principios del derecho internacional. Convirtiendo en positivo el aspecto más espinoso de la ley, reflejando los puntos de vista de sus más decididos defensores, los abogados Alvarez Mena y Daniel Crane reconocen que la ley siempre tuvo la intención de aplicarse extraterritorialmente, ya que persigue a "cualquier persona" que "trafique" con propiedad confiscada. Los apartados más importantes de su análisis son los siguientes:

- Al tiempo que dudan que la Constitución de los Estados Unidos imponga límites a la extraterritorialidad, y recuerdan que el Noveno

${ }^{12}$ Véase el cuadro 2 ofrecido por Ritter.

${ }^{13}$ Véase su trabajo mencionado anteriormente. 
Circuito judicial ha declarado algunos límites, aducen que la mayoría de los casos que se presentaran en los tribunales cumplirían con los requisitos impuestos.

- Con respecto a la compensación por los daños que se reclaman, señalan que deben estar sujetos a un proceso justo para que no resuiten irrazonables o excesivos.

- Con respecto al trato preferencial de ciudadanos norteamericanos (en contraste, por ejemplo, no se protege a los antiguos ciudadanos españoles -que no se convirtieron en norteamericanos- cuyas propiedades fueron confiscadas por la Revolución), se señala que este trato de favor es normal en toda legislación.

- En cuanto al plazo de espera de dos años impuesto a los individuos que no eran ciudadanos de los Estados Unidos en el momento de la confiscación, los autores predicen que no corre peligro de ser denunciado como discriminación étnica (por parte de los mismos ciudadanos norteamericanos de origen cubano).

- Sobre la posible interferencia en la autoridad del Presidente para conducir la política exterior, se recuerda que éste es un caso diferente de la War Powers Act, ya que la ley Helms-Burton trata solamente de temas en los que el Congreso y el Presidente comparten poderes. Mientras el Presidente tiene el poder de administrar la política exterior, no tiene el monopolio para formularla.

- En conclusión, reconocen que la ley puede ser controvertida, pero no se aleja substancialmente de otros precedentes federales.

Por su parte, Brice Clagett considera lo siguiente:

- La intervención de los Estados Unidos está justificada porque las víctimas de las confiscaciones son ahora ciudadanos norteamericanos.

- Debido a la proximidad geográfica de Cuba a los Estados Unidos y la historia entre los dos países, la persistencia cubana en la supresión de la democracia, en la violación de los derechos humanos, y su rechazo a satisfacer la ley internacional tienen efectos en los Estados Unidos, tales como las crisis producidas por las oleadas de refugiados (como el caso espectacular del Mariel en 1980 y los balseros en los noventa). De ahí que los Estados Unidos tengan legítimos intereses para que estos problemas desaparezcan y por ello se justifican las medidas apropiadas y proporcionadas para desincentivar las inversiones en propiedades que tienen un origen "manchado". 
- Teniendo en cuenta la debilidad y la ineficacia de los mecanismos de ejecución de acuerdos internacionales, y a la vista de que la jurisdicción de los tribunales internacionales es consensual, los casos de confiscación raramente llegan al foro apropiado y las acciones gubernamentales se pueden demorar extremadamente (en algunos casos hasta generaciones) hasta que tengan frutos.

- Las leyes internacionales y los derechos humanos reconocen que, en determinadas circunstancias, un Estado puede violar el derecho internacional cuando confisca propiedades tanto de extranjeros como de sus ciudadanos; el título III no se basa en la premisa de que el derecho internacional en todo caso prohibe la confiscación de propiedad de sus ciudadanos sin compensaciones justas.

- El título III de la ley no es injusto con los "traficantes", ya que éstos están plenamente conscientes de que están tratando con propiedades "manchadas" y bienes "robados" y, por lo tanto, están corriendo un riesgo.

\section{Impacto y consecuencias.}

Las repercusiones de la ley Helms-Burton han sido más notables como interposición de un motivo de enfrentamiento entre socios comerciales y aliados políticos.

\section{Reverberaciones americanas.}

Debido a la naturaleza de los existentes acuerdos comerciales de los Estados Unidos, básicamente las reacciones ante la ley se subdividen entre las generadas con sus socios del TLC (NAFTA) y con el resto del continente americano. Las polémicas surgidas con Cuba no son objeto de este estudio.

La ley ha impelido a Canadá a reforzar su política de cooperación en el hemisferio occidental y a reafirmar su consistente política comercial con La Habana, con el resultado de que se ha convertido en el mayor socio comercial de Cuba, solamente superado por Italia en turismo. Desde el triunfo de la Revolución Cubana, la actitud del Canadá hacia Cuba ha estado presidida por tres ejes: pragmatismo diplomático y reconocimiento de un gobierno establecido (aunque no sea del agrado de los canadienses), deferencia tradicional hacia Washington (sobre todo cuando ha gobernado en Ottawa un político más afín a los republicanos), determinación de coexistir con Cuba y al mismo tiempo buscar una 
relación bilateral mutuamente ventajosa. ${ }^{14}$ Como medida más explícita de oposición a la ley Helms-Burton, Canadá aprobó la ley llamada Foreign Extraterritorial Measures Act (FEMA) para evitar que las compañías se plieguen a las exigencias norteamericanas.

Como resultado de las consultas formales celebradas el 28 de mayo de 1996 entre los socios en el TLC, México y el Canadá declararon que la aplicación de la ley "violaba el Derecho internacional" y "podría contravenir las obligaciones del TLC". México advertía que tomaría todas las medidas a su alcance. ${ }^{15} \mathrm{El}$ congreso mexicano emitió un Punto de Acuerdo al día siguiente en el que se destacan los siguientes aspectos:

- la ley "violenta la soberanía" mexicana,

- "castiga a los países que no comparten la política exterior del gobierno estadounidense con respecto a Cuba, situación que es contraria a la tradición de la política exterior mexicana fundada en los principios de no intervención y autodeterminación de los pueblos",

- es "atentatoria al Derecho Internacional al proponer una aplicación extraterritorial",

- México "reitera la solidaridad con el pueblo cubano",

- demanda establecer los mecanismos que defiendan los intereses comerciales de México,

- insta a considerar la "conveniencia de que el gobierno mexicano realice acciones conjuntas cor otros países". 16

Simultáneamente, el gobierno mexicano había manifestado al gobierno norteamericano que la carta de advertencia enviada al grupo Domos (que se debía hacer cargo de la red telefónica, antiguamente administrada por ITT) constituía un intento indebido de hacer valer en México jurisdicción extraterritorial de los Estados Unidos. Más tarde México solicitaba que se reuniera la Comisión del Tratado de Libre Comercio de América del Norte (TLCAN) a la vista de los lineamentos del título IV de la ley, y reiteraba que representaba una amenaza contra las empresas que comercian con terceros países. Reiteradamente México se ha referido al artículo 2006(3) y al 1105 para recordar al gobierno de los Estados Unidos que la ley no debiera afectar a las transacciones protegidas en el TLC ni representar límites de viaje a los ciudadanos

\footnotetext{
${ }^{14}$ Para una revisión adecuada de las relaciones entre Canadá y Cuba se recomiendan los trabajos de Johin Kirk mencionados en la bibliografia.

${ }^{15}$ Comunicado de la Secretaria de Relaciones Exteriores, 28 de mayo de 1997.

${ }^{16}$ Punto de Acuerdo del Congreso de la Unión, 29 de mayo de 1996.
} 
mexicanos. ${ }^{17}$ En una pormenorizada declaración, México resumía su posición:

- México rechazó las sanciones impuestas a Cuba en la OEA y ha condenado también el bloqueo económico impuesto unilateralmente a Cuba, toda vez que el derecho internacional prohibe la imposición de medidas coercitivas.

- Frente a la ley Helms-Burton, México ha expresado su más vigoroso rechazo, pues a la violación de las normas de derecho internacional por la adopción de medidas coercitivas contra el estado cubano, se suma la pretensión de sancionar a personas físicas y morales de nacionalidad mexicana por llevar a cabo operaciones financieras $y$ comerciales en Cuba.

- México ha expresado que continuará haciendo uso de todos los medios legales a su alcance, para impugnar la validez de los aspectos extraterritoriales y contrarios al derecho internacional de esa legislación.

- El recrudecimiento de las sanciones y el aislamiento impuestos a Cuba no han contribuido a incidir o a apoyar cambios en la política interna o exterior del gobierno cubano.

- México reitera su postura en favor del inmediato levantamiento del bloqueo a Cuba, apoya la plena inserción de Cuba en los principales foros regionales y del sistema interamericano, reafirma su posición de que la evolución política y democrática de Cuba es de la incumbencia exclusiva del pueblo cubano. 18

El 23 de octubre de 1996 se publicaba en el Diario Oficial mexicano el texto de una Ley de Protección al Comercio y la Inversión de Normas Extranjeras que contravengan el derecho internacional. Sus aspectos más destacables son los siguientes:

- El artículo 1 prohibe "realizar actos que afecten el comercio o la inversión, cuando tales actos sean consecuencia de los efectos extraterritoriales de leyes extranjeras". Entre estos efectos se mencionan "imponer un bloqueo económico", "permitir reclamar pagos a particulares con motivo de expropiaciones realizadas en el país al que se aplica el bloqueo", "restringir la entrada al país que expide la ley".

- El artículo 2 prohibe proporcionar cualquier información requerida por tribunales a autoridades extranjeras.

${ }^{17}$ Véase el trabajo de Ruiz Bravo.

${ }^{18}$ Posición de México sobre la ley Helms-Burton y la cuestión de Cuba, 28 de agosto de 1996. 
- El artículo 3 conmina a informar al gobierno mexicano sobre los casos de perjuicios y notificaciones mencionados anteriormente.

- El artículo 4 autoriza a los tribunales de México a denegar el reconocimiento y ejecución de sentencias u otros actos jurídicos emitidos con base a las leyes extranjeras referidas, además de reconocer que los condenados al pago de indemnizaciones por sentencias con base en las leyes extranjeras mencionadas tendrán derecho a demandar a su vez en los tribunales mexicanos el pago correspondiente en concepto de daños y perjuicios.

En el seno de la Organización de las Naciones Unidas, México solicitó una opinión consultiva de la Corte Internacional de Justicia y en la Asamblea General se contempló una resolución sobre la aplicación extraterritorial de leyes. En el marco de la OEA, México se sumó a la resolución opuesta a la ley Helms-Burton en el período ordinario de sesiones celebrado en Panamá el 3 de junio de 1996. Allí se tomó nota de la declaración de los ministros de Relaciones Exteriores del Grupo de Río reunidos en Bolivia, con la instrucción al Comité Jurídico Interamericano para que presentara una opinión al Consejo permanente sobre la validez conforme a derecho internacional de la ley. Los puntos más destacados de la Opinión emitida por el Comité Jurídico son los siguientes:

- La ley Helms-Burton no se conforma al derecho internacional pues los tribunales nacionales de un Estado [en este caso, Estados Unidos] no son el foro competente para la resolución de reclamaciones de Estado a Estado, porque [Estados Unidos] no tienen derecho de arrogarse reclamaciones de personas que no eran sus nacionales en el momento de ocurrir el daño, porque no tiene el derecho de atribuir responsabilidad a nacionales de terceros estados por una reclamación que tuviere en contra de otro Estado o por la utilización de bienes expropiados situados en el territorio del Estado expropiante, porque no tiene el derecho de estatuir una responsabilidad de terceros involucrados en una nacionalización, y porque no tiene derecho de imponer compensación por cualquier monto que exceda el daño efectivo.

- En cuanto a la extraterritorialidad de la ley, se recuerda que tales medidas incurren en responsabilidad ya que las normas del derecho internacional no permiten ejercer poder en forma alguna en el territorio de otro Estado, con la única excepción de que un acto ocurrido 
fuera del territorio tenga un efecto directo, sustancial y previsible dentro de su territorio.

- En cuando al "tráfico" de propiedad confiscada, no se conforma con las normas que el derecho internacional establece.

- En resumen, la conclusión establece que los fundamentos y la eventual aplicación de esta ley no guardan conformidad con el derecho internacional. ${ }^{19}$

En el resto de América Latina, ${ }^{20}$ a pesar de las progresivas críticas hacia Castro, la ley ha permitido que numerosos gobernantes recordaran que el mantenimiento de vínculos comerciales es el sistema más idóneo para favorecer la apertura política, sobretodo cuando la amenaza de la infiltración ideológica o a través de las guerrillas es una reliquia del pasado. El Grupo de Río en su décima reunión de jefes de estado y de gobierno celebrada en Cochabamba los días 3 y 4 de setiembre de 1996, al tiempo que no hacía mención sobre la necesidad de evolución democrática en Cuba, como en anteriores ocasiones, incluía una explícita declaración de oposición contra "los efectos territoriales de las leyes nacionales". Se rechazaba cualquier intento de "imponer" sanciones unilaterales que tengan efectos extraterritoriales", porque "violan la ley que regula la coexistencia entre los estados" " "ignora el principio básico del respeto a la soberanía, además de violar el derecho internacional". Por lo tanto, los países miembros del Grupo de Río reafirmaron su "fiuerte rechazo" a la ley, al tiempo que recalcaban la opinión unánime del Comité Jurídico Interamericano de la OEA, referente al detalle de que "la eventual aplicación de la ley no conformaría con el derecho internacional". 21

\section{Entre Washington, Madrid y Bruselas.}

La aprobación de la ley Helms-Burton coincidió con el inminente relevo del gobierno español como resultado de las elecciones celebradas en marzo de 1996. La naturaleza conservadora del nuevo gobierno de José María Aznar hacía predecir una actitud más crítica hacia Castro. Pero el panorama global era más complicado. En el escenario internacional,

\footnotetext{
${ }^{19}$ Opinión del Comité Juridico Interamericano en cumplimiento de la Resolución AG/DOC.337/96 de la Asamblea General de la OEA, titulada "Libertad de Comercio e Inversión en el Hemisferio", 23 de agosto de 1996.

${ }^{20}$ Como muestra reciente del interés que la ley ha despertado en el resto del continente, véase el trabajo de Maria Soledad Torres Macchiavello incluido en la bibilografia.

${ }^{21}$ Declaración de la sesión del Grupo de Río celebrada en Cochabamba, Bolivia. Texto publicado por el Ministerio de Comunicación Social de Bolivia, La Paz, 6 de septiembre de 1996.
} 
Madrid era un causante importante, por sus inversiones y tratos comerciales, del desarrollo de la ley Helms-Burton. El tema de Cuba se había convertido en prácticamente la única desavenencia entre Madrid y Washington, antes y después de los gobiernos socialistas. ${ }^{22}$ Además, en el contexto estrictamente español, Cuba era la causa de una disputa interna entre gobierno y oposición. La ley había obrado un milagro casi irrepetible en el panorama político español. Todos los principales partidos políticos estaban por primera vez de acuerdo en un tema. El PP en el poder y proclive a la libre empresa se aliaba al PSOE e Izquierda Unida (que frecuentemente acosa a ambos), en su oposición a la ley HelmsBurton.

Después de los intercambios verbales entre Castro y Aznar en el contexto de la Cumbre Iberoamericana celebrada en Chile en noviembre de 1996, España presentó ante el Consejo de Ministros de la Unión Europea un nuevo plan de endurecimiento de las relaciones con Cuba ofreciendo que los acuerdos de cooperación mejorarían según fueran las actuaciones cubanas en el terreno político. ${ }^{23}$ Como consecuencia íntimamente relacionada con esta decisión se produjo uno de los incidentes más graves de las nuevas relaciones entre España y Cuba desde el cambio de gobierno en Madrid: la retirada del placet al embajador José Coderch.

La ley ha causado notables brechas en las relaciones interatlánticas, en una etapa inoportuna cuando cualquier desacuerdo comercial puede desencadenar un serio enfrentamiento en la reestructuración de bloques económicos. Con su latente amenaza, aunque suspendida temporal y parcialmente, la Unión Europea decidió denunciarla en el seno de la nueva Organización Mundial del Comercio. Además, Bruselas se apresuraba a aclarar que no retrasaría sus acciones hasta después de las elecciones norteamericanas. Durante la segunda mitad de 1996, el gobierno norteamericano ejerció considerables esfuerzos para tratar de convencer a los miembros de la Unión Europea para que ofrecieran una salida elegante, sobre todo en lo que se refería a las declaraciones de presión o la imposición de condiciones con respecto al proceso de democratización de Cuba. Los gobiernos europeos, sin embargo, se iban a atar las manos con una nueva medida aprobada por el Consejo en noviembre y no podían quedar en evidencia de que negociaban bajo la amenaza de represalias. El Parlamento y la Comisión ya habían emitido

\footnotetext{
${ }^{22}$ Remito a algunos de los estudios propios sobre el tema general de las relaciones entre España y Cuba, cuyos datos completos se incluyen en la bibilografia.

${ }^{23}$ Las lineas generales fueron aprobadas por el Comité Polfitico reunido el 15 de noviembre en Dublin bajo la presidencia irlandesa.
} 
suficientes protestas ${ }^{24}$ y ahora le tocaba el turno (por iniciativa española) al Consejo de la Unión Europea para contrarrestar las acciones de la ley norteamericana.

El mecanismo usado fue un Reglamento aprobado el 22 de noviembre de $1996^{25}$ contra la aplicación de la ley norteamericana. El texto contiene medidas protectoras concernientes sobre la prohibición de aceptar los efectos extraterritoriales de la ley Helms-Burton. En primer lugar, el Consejo establece su justificación para oponerse a la ley y otras medidas similares. La Unión Europea tiene (desde la fundación de la Comuniclad Europea, su predecesora) como uno de sus objetivos contribuir al "desarrollo armónico del comercio mundial y a la abolición progresiva de las restricciones del comercio internacional" $\mathrm{y}$ anhela conseguir al máximo "el libre movimiento de capital entre los estados miembros y terceros países", incluyendo la desaparición de restricciones a las inversiones directas. De acuerdo con estas metas, el reglamento tiene estos principales objetivos:

- Los Estados Unidos han aprobado leyes [Torricelli y Helms-Burton]26 que pretenden regular las actividades de personas bajo la jurisdicción de los estados miembros de la Unión Europea; esta aplicación extraterritorial viola el derecho internacional y tiene efectos adversos para los intereses de la Unión Europea.

- Por lo tanto, el Reglamento proporciona protección contra la aplicación extraterritorial de las leyes y obliga a los afectados a informar a la Comisión.

- Ninguna sentencia de los tribunales ajenos a la Unión Europea con respecto a estas leyes será reconocida y persona alguna deberá cumplir con sus requisitos y prohibiciones.

- Cualquier persona afectada tendrá derecho a ser compensada por los daños causados por la aplicación de estas leyes.

Obsérvese que un repaso comparativo de las medidas canadienses, mexicanas y europeas revela un patrón común repleto de similaridades.

$\mathrm{El}$ inexorable camino de las acciones y reacciones entre Washington y Bruselas desembocó el 3 de febrero de 1997 en la definitiva presentación de una demanda contra los Estados Unidos en el seno de la casi

\footnotetext{
${ }^{24}$ Para un repaso detallado y comparativo, se recomienda la compilación de IRELA, EuropaAmérica Latina: 20 años de documentos oficiales (1976-1996), Madrid, 1996.

${ }^{25}$ Council Regulation (EC), No2271/96, Official Journal of the European Communities, 29 de noviembre de 1996.

${ }^{26}$ Un anexo específicamente menciona todas las medidas legales de los Estados Unidos que la Unión Europea considera inaceptables. Inciuye la "Iran and Lybia Sanctions Act of 1996".
} 
recién nacida Organización Mundial del Comercio (OMC, sucesora del GATT). Era el desenlace de los sucesivos anuncios, a la espera de una rectificación norteamericana que no llegaría. La Unión Europea había advertido que la suspensión temporal del título III no era suficiente, ya que el resto de la ley se consideraba que violaba los principios del intercambio comercial garantizado por la OMC. E1 3 de mayo de 1996 se había emitido una solicitud de celebración de consultas al respecto. Al no haber solución en el horizonte, el 3 de octubre del mismo año el Consejo de Ministros de exteriores de la UE decidió interponer un recurso ante la OMC contra la ley Helms-Burton. El Organo de Solución de Controversias decidió el 20 de noviembre, en aplicación de la reglamentación de la organización, la constitución de un panel para que en el plazo de seis meses emitiera un dictamen. Los Estados Unidos declararon que el tema no era de la competencia de la OMC, pues las limitaciones impuestas a las relaciones comerciales con Cuba eran cuestión de seguridad nacional, ajenas a la jurisdicción puramente comercial de la OMC. Conviene reparar en este detalle con insistencia, ya que confirma el objetivo político de la ley.

\section{3. ¿Muerte y resurrección de la ley?}

Apenas rebasado el primer aniversario de la ley Helms-Burton, se produjo el anuncio de un compromiso entre la Unión Europea y los Estados Unidos para poner fin a los enfrentamientos justamente en la víspera de que comenzaran las acciones en el marco de la OMC. El 11. de abril de 1997, un día antes de cumplirse trece meses de la aprobación de la ley, la Unión Europea anunciaba "un acuerdo de principio" con Estados Unidos que conduciría el 25 de abril a la suspensión de los trabajos del panel. Se intuía que los Estados Unidos debieran haberse comprometido a alguna medida a cambio de que la Unión Europea retirara su denuncia y además se comprometiera a poner en marcha ciertas medidas "desalentadoras" de las inversiones en Cuba. Por otra parte, el presidente Clinton anunciaba que la política general hacia Cuba no cambiaría. ${ }^{27}$ La Casa Blanca y el Departamento de Estado, por otra parte, eran conscientes de que la demanda interpuesta ante la OMC era un callejón sin salida que abriría la puerta a daños colaterales en todo el entramado del libre comercio que se está negociando.

${ }^{27}$ Cables de AP, EFE, y otras agencias, 11 de abril de 1997. El 18 de abril el COREPER ratificaba el acuerdo. 
Los detalles de la oferta de los Estados Unidos incluían seguir suspendiendo indefinidamente el título III de la ley y persuadir al Congreso para congelar el título IV que deniega visados a los dirigentes de las compañías que trafiquen en propiedades confiscadas; la Unión Europea, a cambio, se comprometía a acordar medidas que limitaran (desaconsejaran) los tratos comerciales en tales propiedades. Ambas partes acordaban trabajar conjuntamente en promover la democracia en Cuba. ${ }^{28}$ Los dos protagonistas del acuerdo reclamaban que habían logrado beneficios mutuos y ventajas para sus propios intereses. Brittan consideraba como triunfos, a cambio de haber retirado la demanda ante la OMC, los siguientes: que el acuerdo también incluyera la protección de inversiones en otras regiones (como Libia e Irán), que se limitara a inversiones futuras (no a las que ya están en manos de intereses europeos), y que neutraliza este tipo de leyes de aplicación extraterritorial. En sus declaraciones diseminadas por distintos comunicados de prensa, Eizenstat se congratulaba de haber evitado un daño a la $O M C$, mediante el acuerdo que "crea la primera y verdaderâ oportunidad para desarrollar disciplinas multilaterales que impidan y prohiban invertir en propiedades confiscadas", y que "establece fuertes normas globales para acrecentar la protección de los derechos de propiedad". 29 Los observadores europeos ya tempranamente habían expresado su preocupación sobre las dificultades que debería enfrentar el presidente Clinton en el Congreso.

Cuando en julio de 1997 el presidente Clinton de nuevo decidió suspender el título III de la ley, el representante Bill McCollum (republicano por Florida) resolvió presentar un proyecto de ley que despojaría al presidente de esta prerrogativa. Se aducía que no se habían cumplido dos condiciones fundamentales para justificar una nueva congelación transitoria: el interés nacional de los Estados Unidos y alguna evidencia de que había comenzado la transición política en Cuba.

En conclusión, la suspensión de los títulos III (por decisión del presidente) y IV (por consenso con el Congreso) hubiera significado que la ley quedaría virtualmente sin contenido, aunque la Unión Europea y los Estados Unidos declararan que se reservaban sus opciones a la vista de los respectivos movimientos. Este desenlace confirmaría la tesis de que las diversas medidas de cada una de las partes se considerarían como

\footnotetext{
28 Proyecto de Acuerdo (Memorandum of Understanding), 11 de abril de 1997.

29 "Enfoque multilateral a los derechos de propiedad", comunicado transcrito en versión en español en Diario las Américas, 27 de abril de 1997.
} 
secundarias para evitar el enfrentamiento directo entre la Unión Europea y los Estados Unidos con consecuencias graves para el comercio mundial. El límite ha sido el posible e irreparable daño causado a la Organización Mundial del Comercio; en la triangular relación entre los Estados Unidos, Canadá y México, el límite está constituido por la fragilidad del TLC.

El tema queda abierto y deberá ser analizado no solamente como mínimo en el plazo de los seis meses propuestos como una especie de tregua, sino también durante otro período de medio año después de la nueva suspensión del título III acordada por Clinton y en vista de las nuevas endurecedoras medidas del Congreso. En cualquier caso, de cumplirse todos los puntos del acuerdo entre los Estados Unidos y la Unión Europea, si el actual régimen cubano termina por la simple desaparición de Fidel Castro, la ley Helms-Burton pasará a la historia con un balance de deudas mayor que el de beneficios, ya que el objetivo central (la desaparición del presente régimen cubano bajo presión económica) no se habrá conseguido. El daño inflingido a las relaciones interatlánticas y el debilitamiento que habrá recibido el entramado jurídico del comercio mundial (principalmente la OMC) constituyen un lamentable legado. ${ }^{30}$ La ley Helms-Burton quedará simplemente como testimonio de un intento por acelerar el resurgimiento de la democracia en Cuba (meta final compartida por todas las partes) y como una innecesaria desavenencia entre los Estados Unidos y el resto del mundo.

\section{Bibliografía}

Altozano, Hermenegildo, "La Ley Helms-Burton y la protección de las inversiones españolas en Cuba", en Seminario sobre "El refuerzo del embargo de EE.UU. contra Cuba", Sitges, 8-10 de julio de 1996.

Alvarez-Mena, Sergio, y Daniel A. Crane, "From the commerce clause to café cubano: the constitutionality ofHelms-Burton", Ponencia, American Bar Association International Law Section and Inter-American Law Committee, Fall Meeting, 23-26 de octubre de 1996.

Bar Association of the City of New York. The Committee on Inter-American Affairs, "The legality of the extraterritorial reach of the Cuban Democracy Act of 1992", The Report, May-June 1966.

${ }^{30}$ Se recomienda la consulta del lúcido artículo de Kinka Gerke, mencionado en la bibliografia. 
Bourque, Shari-Ellen, "The Illegality of the Cuban Embargo in the Current International System", Boston University International Law Journal, Vol. 13:163, Spring 1995, pp.191-228.

Cain, Jerry W. Jr., "Extraterritorial Application of the United States' Trade Embargo Against Cuba: The United Nations Geveral Assembly's Call for an End to the U.S. Trade Embargo", Georgia Journal of International Law and Comparative Low, N2, 1994, pp. 379-396.

Clagett, Brice M., "Title III of the Helms-Burton Act is Consistent with Intemational Law", The Americar Journal of International Law, Vol. 90, №3, July 1996, pp. 434-440.

De la Cámara, Manuel, "Lã ley Helms-Burton sobre embargo a Cuba", Información Comercial Española, Boletín Económico del ICE, 2502, 27 de marzo-2 de junio de 1996, pp. 23-32.

Donner, Laura A., "The Cuban Democracy Act of 1992: Using Foreign Subsidiaries as Tools of Foreign Economic Policy", Emory International Review, Vol. 7, N¹, Spring 1993, pp. 259 267.

Estados Unidos, Congressional Record-Senate, October 24, 1995. -- - Congressional Record-House, September 21, 1995.

Estados Unidos. U.S. Congress, "Ley de la Libertad y la Solidaridad con Cuba", versión en español de la oficialmente denominada "Cuba Liberty and Democratic Solidarity (Libertad) Act of 1996", Pub. I, N¹04-114, 110 Stat. 785, 1996.

- - - U.S. Congress, Cuban Liberty and Democratic Solidarity Act: Hearings Before the Subcommittee on Western Hemisphere and Peace Corps Affairs of the Senate Committee on Foreign relations, 104th Congress lst Session (1995).

Gerke, Kinka, "The Transatlantic Rift over Cuba. The Damage is Done", The International Spectator, XXXII, 2, April-June 1997, pp.27-61.

Hernández Truyol, Berta Esperanza, "Out in left field: Cuba's post-cold war strikeout", Fordham International Law Journal, 18, $\mathrm{N}^{\circ} 1$, November 1994, pp.15-117.

IRELA, "La posición común de la UE sobre Cuba: debate interno, reacciones y repercusiones", Informe, Madrid, 13 de diciembre 1996.

--- , "Cuba y la Unión Europea: las dificultades del diálogo", Informe, 17 de junio de 1996.

- - - -, "Europa-América Latina: 20 años de documentos oficiales (19761996)", Madrid, 1996.

Kaplowitz, Donna Rich, "El comercio a través de filiales entre Estados Unidos y Cuba: antes y después de la 'Cuban Democracy Act' ", Estudios Internacionales, $\mathrm{N}^{\circ} 107-108,1994$, pp. 469-495. 
Kirk, John M., "Descifrando la paradoja: la posición del Canadá respecto de Cuba", Estudios Internacionales, N0107-108, 1994, pp. 570-585.

Kirk, John M., A la redécouberte de Cuba: Cinquante années de relations canado-cubaines. Ottawa: Les Cahiers de FOCAL, 1997.

Kirk, John M. and Peter McKenna, Canada-Cuba Relations: the other Good Neighbor Policy. Gainesville, Fla.: University Press of Florida, 1997.

Kneale, John, "Tightening the US embargo: talking points", en seminario sobre "El refuerzo del embargo de EE.UU. contra Cuba", Sitges, 8-10 de julio de 1996.

Krinsky, Michael, y David Golove (eds.), United States Measures Against Cuba. Proceedings in the United Nations and International Law Issues. Northampton, Ma.: Aletheia Press, 1993.

Kourliandsky, Jean-Jacques, "Cuba: Un embargo 'mère' des sanctions économiques contemporaines", Relations internationales et stratégiques, 24, hiver 1996, pp.172-180.

Leogrande, William M., "Enemies Evermore: US Policy Towards Cuba after Helms-Burton", Journal of Latin American Studies, Vol. 29, No1, pp.211-221.

Lisio, Stephen A., "Helms-Burton and the Point of Diminishing Returns", International Affairs, Vol. 72, N2, pp. 691-711.

Lowenfeld, Andreas F., "The Cuban Liberty and Democratic Solidarity (Libertad) Act", The American Journal of International Law, Vol. 90, N³, July 1996, pp.419-434.

Mallett, Nick, "The application in the United Kingdom of the Cuban Liberty and Democratic Solidarity Act (libertad) Act of 1996", en seminario sobre "El refuerzo del embargo de EE.UU. contra Cuba", Sitges, 8-10 de julio de 1996.

Meron, Theodor; Vagts, Detlev F., "The Helms-Burton Act: Exercising the Presidential Option", The American Journal of International Law, Vol. $91, N^{\circ} 1, p p .83-84$.

Muse, Robert, "Legal and practical implications of Title III of the Helms-Burton Law", en seminario sobre "El refuerzo del embargo de EE.UU. contra Cuba", Sitges, 8-10 de julio de 1996.

Ondetti, Gabriel A., "Western European and Canadian Relations with Cuba after the Cold Wa"', Cuba Briefing Paper Series, Georgetown University, $\mathrm{N}^{\circ} 9$, November 1995.

Perera Gómez, Eduardo, "Cuba en la estrategia reciente de la Unión Europea", Revista de Estudios Europeos, 1995, 36, pp. 139 145.

- - - " "Cuba and the European Union: factors of Stagnation", Revista de Estudios Europeos, Vol. X, N40, La Habana, October-December 1996, pp. $78-115$. 
Porotsky, Richard D., "Economic Coercion and the General Assembly: a Post-Cold War Assessment of the Legality and Utility of the Thirty-Five Year Old Embargo Against Cuba", Vanderbilt Journal of International Law, Vol. 28, №4, October 1995, pp. 901-958.

Ritter, Archibald R. "El problema de las compensaciones: ¿quién compensa a quién, por qué y cómo?", Estudios Internacionales, N¹07-108, 1994, pp. 496-512.

Robaina, Roberto, "La realidad cubana ante la Unión Europea", Política Exterior, 57, XI, mayo-junio 1997, pp. 87-93.

Roy, Joaquín, "Las relaciones actuales entre Cuba y España", Politica Exterior, Vol. I, No3, verano 1987, pp. 282-286.

- - -, "Las relaciones Madrid-La Habana", Política Exterior, Vol. II, №6, primavera 1988, pp. 275-279.

- - -, "Las relaciones actuales entre Cuba y España", Afers Internacionals, No12-13, Barcelona, 1988, pp. 5-19.

- - - ", "Cuba: des inconnues pour l'avenir", L'Espagne et l'Amérique Latine, Documentation Française, No595, Paris, 11 de noviembre de 1988, pp. 24-28.

----, Cuba y España: relaciones y percepciones. Madrid: Biblioteca Cubana Contemporánea, $1988,100 \mathrm{p}$.

-_--, "Relaciones y percepciones entre España y Cuba. Trasfondo de la 'Crisis de las embajadas" ", en: Carlos Robles Piquer(ed.), Cuba 1990: Realidad y futuro. Santiago de Compostela: Fundación Alfredo Brañas, 1991, pp. $27-47$.

- - - "España y Cuba: Una relación muy especial"; "Posdata"; en: Joaquín Roy y Juar Antonio March (ed.), El espacio iberoamericano: dimensiones y percepciones de la relación especial entre España y América Latina. Miami / Barcelona: Instituto de Estudios Ibéricos/ Centro de Estudios Internacionales, 1996.

-_- "España, la Unión Europea y Cuba: la evolución de una relación especial a una política de gestos y de presión", Occasional paper, University of Miami, Cuban Student Association, Miami, 1996, 42 p.

----, "España y Cuba: una relación muy especial", Revista Afers Internacionals, No31, Barcelona, 1996, pp.147-166.

Ruiz Bravo, Hernán de Jesús, "La Ley Helms-Burton: líneas de acción para contrarrestar sus efectos extraterritoriales", en seminario sobre "El refuerzo del embargo de EE.UU. contra Cuba", Sitges, 8-10 de julio de 1996.

Sabbach, Daniel, "L'utilisation de l'arme économique dans la politique étrangère des Etats-Unis: cadre d'analyse et évolution", Relations internationales et stratégiques, 24, hiver 1996, pp.135-149. 
Smith, Wayne S., "The Helms-Burton Law: Danger Signal for the Future", en seminario sobre "El refuerzo del embargo de EE.UU. contra Cuba", Sitges, 8-10 de julio de 1996.

Unión Europea, Council Regulation (EC), N2271/96, Official Journal of the European Communities, 29 de noviembre de 1997.

Unión Europea, "Comunicación de la Comisión al Consejo y al Parlamento Europeo", 28 de junio de 1995.

Unión Europea. Parlamento Europeo, "Resolución sobre la situación de los presos políticos en Cuba", 29 de octubre de 1987.

- - - Parlamento Europeo, "Resolución sobre las relaciones de la CE con los Estados no europeos, miembros del CAME", 31 de octubre de 1988.

- - - Parlamento Europeo, "Resolución sobre la situación actual en Cuba, particularmente en materia de derechos humanos", 15 diciembre de 1988.

- - - Parlamento Europeo, "Resolución sobre Cuba", 13 de septiembre de 1990.

-- - Parlamento Europeo, "Resolución sobre las ejecuciones y las violaciones de los derechos humanos en Cuba", 13 febrero de 1992.

- - Parlamento Europeo, "Resolución sobre las restricciones al comercio internacional por parte de los Estados Unidos (Cuban Democracy Act)", 17 de diciembre de 1992.

- - - . Parlamento Europeo, "Resolución sobre la situación de los derechos humanos en Cuba", 11 de marzo de 1993.

- - - Parlamento Europeo, "Resolución sobre el embargo contra Cuba y la "ley Torricelli"”, 16 de septiembre de 1993.

- - - Parlamento Europeo, "Resolución sobre la situación en Cuba", 29 de septiembre de 1994.

- - - Parlamento Europeo, "Resolución sobre la Comunicación de la Comisión al Consejo y al Parlamento Europeo sobre las relaciones entre la Unión Europea y Cuba", 18 de enero de 1996.

- - - Parlamento Europeo, "Resolución sobre Cuba", 14 de marzo de 1996.

-- - Parlamento Europeo," Resolución sobre las obligaciones de los Estados Miembros en relación con el GATT", 10 de junio de 1996.

Unión Europea. Consejo, "Declaración sobre Cuba", 23 de enero de 1992.

- - - Consejo, "Declaración sobre Cuba", 22 de diciembre de 1992.

Unión Europea, "Declaración de la Presidencia en nombre de la Unión Europea sobre Cuba", 5 de abril de 1995.

- - - "Declaración de la Presidencia en nombre de la Unión Europea sobre el proyecto de ley Helms-Burton", 11 de octubre de 1995. 
Unión Europea, "Declaración de la Unión Europea sobre la destrucciỏn de dos aviones civiles por las autoridades cubanas", 26 de febrero de 1996.

- - - - Reglamento del Consejo de la Unión Europea N2271/96, Official Journal of the European Communitie, 29 de noviembre de 1997.

United Kingdom's Protection of Trading Interests (U.S. Cuban Assets Control Regulations) Order, 1992, N2449, October 14.

Viñas, Angel, "La Unión Europea y Cuba: historia de una acción de estrategia exterior en la post guerra fría", en: Temas de economía internacional. Bilbao: Universidad del País Vasco, 1996, pp. 311-359.

Wong, Kam S., "The Cuba Democracy Act of 1992: The Extraterritorial Scope of Section 1706(a)", University of Pennsylvania Journal of International Business Law, Vol. 14:4, Winter 1994, pp. 651-682.

Zipper, Arnold M., "Toward the Termination of Licensed U.S. Foreign Subsidiary Trade With Cuba: The Legal and Political Obstacles", Low and Policy in International Business, Vol. 23, No4, 1992, pp. 1045-1069. 